\title{
O conceito do insight em pacientes com transtorno obsessivo-compulsivo
}

\section{The concept of insight in patients with obsessive-compulsive disorder}

\author{
Júlia M. Fontenelle, ${ }^{1}$ Lívia da S. Santana, ${ }^{1}$ Larissa da R. Lessa, ${ }^{1}$ Mara S. da Victoria, ${ }^{1}$ Mauro V. Mendlowicz, ${ }^{2}$ \\ Leonardo F. Fontenelle $\mathrm{e}^{1,2,3}$ \\ 1 Programa de Ansiedade e Depressão, Instituto de Psiquiatria, Universidade Federal do Rio de Janeiro (IPUB/UFRJ), Rio de Janeiro (RJ), Brasil \\ 2 Departamento de Psiquiatria e Saúde Mental, Instituto de Saúde da Comunidade, Universidade Federal Fluminense (MSM/UFF), Niterói (RJ), \\ Brasil \\ 3 Departamento de Psiquiatria e Medicina Legal, Faculdade de Medicina, Universidade Federal do Rio de Janeiro (UFRJ), Rio de Janeiro (RJ), \\ Brasil
}

\begin{abstract}
Resumo
Objetivo: Atualizar os clínicos sobre a existência de um possível subtipo do transtorno obsessivo-compulsivo caracterizado por pouco insight. Método: Revisão opinativa baseada em estudos indexados na base de dados PubMed e PsychINFO, identificados por meio dos unitermos "obsessive-compulsive disorder" e "insight" ou "ego-dystonic" e publicados entre 1966 e outubro de 2009. Os resultados foram examinados de acordo com a estratégia utilizada para abordar o insight, i.e. categórica vs. dimensional. Resultados: Análise dos estudos nos permitiu identificar pontos que colocam em dúvida a existência de um subtipo do transtorno obsessivo-compulsivo bem delimitado caracterizado por pouco insight. Estes pontos incluem 1) prevalência extremamente variável do transtorno obsessivo-compulsivo com insight reduzido encontrada em estudos categóricos, 2) homogeneidade dos achados fenotípicos (i.e. maior gravidade) associados a baixo insight em estudos categóricos e dimensionais e 3) ausência de estudos que investigam "zonas de raridade" entre as formas de transtorno obsessivo-compulsivo com pouco e bom insight. Conclusão: Embora uma abordagem categórica do insight no transtorno obsessivo-compulsivo seja importante em ambientes clínicos, uma vez que neles existem demandas prementes para tomada de decisões, a abordagem dimensional do insight parece refletir de forma mais fidedigna o fenômeno apresentado pelos pacientes em tela.
\end{abstract}

Descritores: Transtorno obsessivo-compulsivo; Psicopatologia; Prevalência; Fenótipo; Tomada de decisóes
Abstract

Objective: To update clinicians regarding the existence of a putative subtype of obsessive-compulsive disorder based on poor insight. Method: Opinionative review based on studies indexed in the PubMed and PsychINFO databases, identified by means of the keywords "obsessive-compulsive disorder" AND "insight" OR "ego-syntonic", and published between 1966 and October 2009. The results were analyzed according to the approach adopted, i.e. a categorical or dimensional view of insight in obsessive-compulsive disorder. Results: The review of recent studies led us to identify some issues that cast doubts over the existence of a clear-cut poor insight subtype of obsessivecompulsive disorder. These issues include 1) an extremely variable prevalence of poor insight obsessive-compulsive disorder in categorical studies, 2) a significant degree of homogeneity in the phenotypical findings (i.e. greater severity) associated with lower levels of insight in obsessive-compulsive disorder in both categorical and dimensional studies and, 3) a lack of studies investigating zones of rarity between poor and good insight obsessivecompulsive disorder. Conclusion: Although a categorical approach to the insight issue in obsessive-compulsive disorder is still important in clinical settings, where decision-making is often a critical issue, a dimensional approach seems to reflect levels of impairment in these patients more reliably.

Descriptors: Obsessive-compulsive disorder; Psychopathology; Prevalence; Phenotype; Decision making
Submetido: 3 Setembro 2009

Aceito: 25 Novembro 2009

\section{Correspondência}

Leonardo F. Fontenelle

Rua Visconde de Pirajá, 547, Sala 719 - Ipanema

22410-003 Rio de Janeiro, RJ, Brasil

Tel./Fax: (+55 21) 2239-4919 


\section{Introdução}

O insight é a capacidade que o ser humano possui de avaliar criticamente seu estado mental com os "olhos da mente", sejam eles os do próprio indivíduo ou os de terceiros ${ }^{1}$. Inclui o conhecimento a respeito de si próprio e a aceitação da existência de uma condição mórbida e da respectiva necessidade de tratamento, quando este é o caso. Classicamente, credita-se aos portadores do transtorno obsessivo-compulsivo (TOC) um bom nível de crítica em relação aos seus próprios sintomas, i.e. um elevado nível de insight. No entanto, existe um reconhecimento crescente de que uma parcela considerável destes pacientes é parcial ou totalmente incapaz de reconhecer a irracionalidade de seus sintomas, ou seja, apresenta pouco ou nenhum insight. Nesses casos, os sintomas apresentados são melhor descritos como ideias supervalorizadas ou delírios, respectivamente $e^{3-6}$.

Com frequência, os termos ego-distônico e ego-sintônico são também utilizados para designar o discernimento crítico dos pacientes sobre seus sintomas. Pensamentos, impulsos, atitudes, comportamentos e sentimentos que contrariam e perturbam a pessoa, e que por isso não estão em sintonia com o ego, são chamados ego-distônicos. Por outro lado, sintomas que não são percebidos pelo paciente como estranhos a si, e.g. sintomas obsessivo-compulsivos de indivíduos com TOC e pouco ou nenhum insight, são denominados também de ego-sintônicos? ${ }^{7}$.

Diversos autores propuseram variadas estratégias para determinar o grau de insight de pacientes com TOC. Estes métodos incluem não apenas uma avaliação clínica, baseada somente nos critérios da DSM-IV (i.e. "insight pobre") de instrumentos específicos, como a Brown Assessment of Beliefs Scale (BABS) ${ }^{9}$, o item 11 da Yale-Brown Obsessive-Compulsive Scale (Y-BOCS) ${ }^{10}$, a Overvalued Ideas Scale (OVIS) ${ }^{11}$ e a Fixity of Beliefs Scale (FBS $)^{12}$. Nestes estudos, duas formas de abordagem do insight têm sido adotadas. Uma delas é categórica, considerando o insight como um fenômeno ou presente ou ausente e dividindo os pacientes com TOC naqueles com muito ou pouco insight. A outra é dimensional, admitindo que o insight é um atributo que pode variar de ausente a presente gradativamente, com diferentes níveis possíveis entre os dois extremos.

No presente estudo, pretendemos rever os trabalhos que avaliaram o insight em pacientes com TOC na tentativa de confirmar ou refutar a existência de um subtipo do TOC bem delimitado e caracterizado por pouco insight. Para isso, procuramos responder às seguintes perguntas:

1) Qual a prevalência do subtipo de TOC caracterizado por pouco insight? Caso exista, este subtipo deve se apresentar com uma frequência relativamente constante em estudos que utilizaram a abordagem categórica do insight em amostras clínicas com características sociodemográficas comparáveis.

2) Quais são os achados fenotípicos do subtipo de TOC com pouco insight? Se este subtipo existe, deve se caracterizar por um fenótipo qualitativamente bem definido em estudos que utilizaram a abordagem categórica do insight, em contraposição àqueles que utilizaram a abordagem dimensional ao referido construto.

\section{Método}

Realizamos uma revisão opinativa baseada em estudos indexados na base de dados PubMed e PsychINFO, identificados através dos unitermos "obsessive-compulsive disorder" e "insight" ou "egodystonic" e publicados entre 1966 e outubro de 2009. Somente estudos originais em inglês, francês, espanhol ou português foram selecionados. Os estudos foram examinados e classificados de acordo com a estratégia utilizada para abordar o insight, i.e. categórica ou dimensional.

\section{Resultados}

Inicialmente, nossa estratégia de busca permitiu identificar 183 estudos no PubMed e 151 estudos no PsychINFO. Estes trabalhos foram avaliados criticamente por dois autores, que selecionaram somente estudos originais sobre correlatos sociodemográficos, clínicos, biológicos e evolutivos do insight em pacientes com TOC. Apenas dois estudos identificados no PsychINFO não se encontravam indexados no PubMed. Os estudos selecionados foram então divididos naqueles que abordaram o insight de forma categórica $(\mathrm{n}=18)$ ou dimensional $(\mathrm{n}=23)$, conforme discutiremos a seguir.

\section{Insight como variável categórica}

Aqueles estudos que classificaram o insight como presente ou ausente identificaram diferentes frequências de ausência desta habilidade em pacientes com TOC, variando de $4 \%{ }^{12}$ a $36 \%{ }^{10}$ e incluindo uma ampla faixa de valores intermediários: $6 \%{ }^{13}$, $15 \%^{14}, 16 \%{ }^{15}, 25 \%^{16}$ e $29,5 \%{ }^{17}$. Em crianças e adolescentes, que apresentam níveis inferiores de desenvolvimento mental, a prevalência de comprometimento do insight foi observada em até $45 \%$ dos pacientes ${ }^{18}$. A heterogeneidade desses dados reflete não apenas diferenças entre as amostras estudadas, mas também critérios distintos para estabelecer o nível de insight.

Em termos clínicos, pacientes com TOC e pouco insight caracterizaram-se por idade de início mais precoce $^{16}$, duração mais longa da doença ${ }^{16}$ e maior número de sintomas obsessivocompulsivos ${ }^{16,19}$. Indivíduos com TOC e pouco insight também apresentaram maior gravidade de sintomas obsessivocompulsivos $^{8,15,19}$, depressivos ${ }^{8,17,19}$ e ansiosos ${ }^{8}$, prevalência mais elevada da lentidão obsessiva ${ }^{20}$, de depressão maior ${ }^{8,16}$ e de transtornos de personalidade borderline ${ }^{8}$, narcisista ${ }^{8}$ e esquizotípico ${ }^{17}$, e menor prevalência de transtorno de personalidade dependente ${ }^{8}$. Em um estudo ${ }^{10}$, adultos com TOC e pouco insight manifestaram um comprometimento funcional semelhante ao de pacientes com esquizofrenia e TOC. Por outro lado, pacientes com TOC e preocupaçôes com a própria saúde exibiram, mais comumente, perda de insight do que pacientes com TOC sem preocupações com a saúde ${ }^{21}$.

No que diz respeito à história patológica pregressa, adultos com TOC e pouco insight apresentaram maior frequência de transtornos psiquiátricos durante a infância ${ }^{15}$. De forma semelhante, e de acordo com a avaliação feita pelos pais, crianças com TOC e pouco insight exibiram maior comprometimento funcional e maior acomodação familiar ${ }^{18}$. Ainda no âmbito doméstico, pacientes 
com TOC e pouco insight caracterizaram-se por maior expressão emocional ${ }^{19}$, maior crítica familiar percebida ${ }^{19}$ e também maior prevalência de transtornos do espectro esquizofrênico em familiares de primeiro grau ${ }^{15}$.

Do ponto de vista neuropsicológico, pacientes com TOC e pouco insight obtiveram um desempenho cognitivo semelhante ao de indivíduos com esquizofrenia ${ }^{22}$. Além disso, uma maior prevalência de alterações no exame de ressonância magnética do crânio foi observada nestes pacientes, em comparação com pacientes com TOC e insight preservado ${ }^{23}$.

Pacientes com TOC e pouco insight no início da doença relataram busca por ajuda terapêutica mais tardiamente do que aqueles pacientes com bom insight $t^{24}$. Finalmente, no que concerne à resposta terapêutica, pacientes com TOC e pouco insight exibiram pior resposta ao tratamento com inibidores de recaptação da serotonina (IRS), combinados ${ }^{10,25}$ ou não ${ }^{15,26,27}$ à terapia cognitivo-comportamental. Apesar destes achados relativamente desanimadores, no estudo de Matsunaga et al. ${ }^{10}, 14 \mathrm{em} 25$ pacientes com TOC e pouco insight não foram mais classificados como tal após seis meses de tratamento combinado e melhora dos sintomas obsessivo-compulsivos e depressivos associados.

\section{Insight como variável contínua}

Uma série de estudos também foi conduzida tratando o insight como variável contínua e dependente, tendo encontrado níveis significativamente reduzidos de insight em associação com diversas variáveis sociodemográficas clínicas e evolutivas.

A gravidade de diferentes tipos de sintomas, obsessivocompulsivos ou não (como compulsões ${ }^{11}$, depressão ${ }^{19,28} \mathrm{e}$ alexitimi ${ }^{28}$ ) se correlacionou negativamente com os níveis de insight, com poucas exceçôes ${ }^{29}$. O insight de pacientes com TOC correlacionou-se negativamente com cinco dentre sete dimensōes de qualidade de vida medidas pelo Short-Form $36\left(\right.$ SF-36) ${ }^{30}$.

Níveis reduzidos de insight foram observados em adultos com variadas expressōes de sintomas obsessivos. Por exemplo, obsessōes agressivas (incluindo a chamada "fobia de impulso") ${ }^{31}$, religiosas ${ }^{31}$, somáticas $^{14,28}$ e de colecionamento ${ }^{28}$, assim como compulsōes de checagem e de lavagem simultâneas ${ }^{32}$, associaram-se a níveis reduzidos de insight em diferentes estudos. $\mathrm{Na}$ única pesquisa do gênero realizada com crianças, Storch et al. observaram níveis significativamente reduzidos de insight em pacientes com TOC e colecionismo patológico ${ }^{33}$.

De forma semelhante aos trabalhos que utilizaram o insight como variável independente, a presença de uma diversidade de transtornos comórbidos associou-se a níveis menores de insight em pacientes com TOC, incluindo o transtorno dismórfico corporal $^{34-36}$, o transtorno hipocondríaco ${ }^{34}$, os transtornos de personalidade esquizotípico ${ }^{37}$ e obsessivo-compulsivo ${ }^{12,38}$ e a síndrome de Asperger ${ }^{39}$. Em oposição aos estudos que encontraram uma maior prevalência de transtorno de personalidade obsessivocompulsivo em pacientes com TOC e pouco insight, Bellino et al., por razōes ainda desconhecidas, notaram que pacientes com transtorno de personalidade obsessivo-compulsivo caracterizaramse por maiores níveis de insight. Pacientes com outros atributos, como curso crônico e história familiar positiva de TOC, também apresentaram menores níveis de insight ${ }^{11}$.

Um grupo observou que pacientes com TOC e esquizofrenia demonstraram menor insight sobre seus sintomas do que pacientes com TOC em geral ${ }^{10}$. Curiosamente, Ozdemir et al. constataram que pacientes com TOC e esquizofrenia expressaram maior insight em relação aos sintomas obsessivo-compulsivos do que pacientes com TOC sem esquizofrenia ${ }^{40}$. No estudo de Poyurovky et al., a maioria dos pacientes com esquizofrenia e TOC (84,2\%) revelou bom insight em relação aos sintomas obsessivocompulsivos, embora não necessariamente em relação aos sintomas esquizofrênicos ${ }^{41}$. Por outro lado, Marazitti et al.observaram que pacientes com transtorno bipolar demonstravam uma tendência a apresentar maior insight a respeito dos seus sintomas obsessivos do que os demais pacientes com TOC ${ }^{14}$. Esse achado foi confirmado por Tukel et al., que encontraram melhor insight em relação aos sintomas obsessivo-compulsivos em pacientes com TOC e transtorno bipolar do que em pacientes com TOC e depressão maior ou em pacientes com TOC sem transtornos do humor ${ }^{42}$.

No que se refere à resposta terapêutica, níveis reduzidos de insight pré-tratamento foram observados em adultos que não responderam aos IRS, potencializados por risperidona ${ }^{43}$ ou não ${ }^{16}$, e à terapia cognitivo-comportamental em grupo ${ }^{44}$. Este mesmo fenômeno foi constatado em crianças refratárias a diversas formas de tratamento ${ }^{18}$. Divergindo dos estudos descritos até o momento, Eisen et al. notaram que o grau de insight não foi capaz de predizer a resposta à sertralina, i.e. pacientes com diferentes níveis de insight responderam ao tratamento medicamentoso da mesma maneira ${ }^{45}$.

\section{Discussão}

A análise dos estudos sobre insight de acordo com o tipo de abordagem adotada (categórica vs. dimensional) nos permitiu identificar alguns achados que colocam em dúvida a existência do TOC com pouco insight como subtipo bem delimitado. Estes achados incluem 1) uma prevalência extremamente variável do TOC com insight reduzido nos estudos categoriais, 2) uma maior gravidade (seja em termos de sintomas, múltiplas comorbidades ou prejuízo psicossocial) em associação ao insight reduzido, tanto nos estudos categoriais quanto nos dimensionais e 3) uma ausência de características exclusivas do TOC com insight reduzido em diferentes estudos. Neste sentido, a estratégia adotada pela Diagnostic and Statistical Manual, Fourth Edition, Text Revision (DSM-IV-TR), que busca identificar um subtipo do TOC de pouco insight bem delimitado, nos parece pouco frutífera, ao menos em termos de sua caracterização fisiopatológica. Admitimos, no entanto, que em ambientes clínicos, quando existem demandas prementes para tomada de decisões (e.g. empreender ou não um tratamento específico), a abordagem categórica ainda se faz indispensável. De qualquer forma, os achados que suscitam dúvidas sobre a existência do TOC com pouco insight como subtipo categoricamente bem definido merecem ser examinados com detalhes.

Em primeiro lugar, estudos categoriais que classificaram o insight como reduzido identificaram diversas frequências do fenômeno, 
que acomete desde $4 \%{ }^{12}$ até $36 \%{ }^{10}$ dos pacientes adultos. Como se pode supor, a heterogeneidade desses dados reflete a adoção de diferentes instrumentos (i.e. a DSM-IV-TR ${ }^{8}$, o $\mathrm{BABS}^{9}$, o item 11 da Y-BOCS ${ }^{10}$, a OVIS ${ }^{11}$ e a FBS ${ }^{12}$ ) e de distintos pontos de corte para classificar o nível de insight, uma limitação inerente à abordagem categórica deste construto. No entanto, é possível que descrições sistemáticas das frequências com que são observadas ausência total (i.e. delírios) ou parcial (i.e. ideias supervalorizadas) do insight em estudos futuros com subgrupos específicos de pacientes com TOC (e.g. amostras clínicas de pacientes com tiques ou com início precoce dos sintomas) possam gerar números mais consistentes.

De fato, o próprio conceito de insight no TOC tem variado consideravelmente, a começar por seu próprio objeto ${ }^{46}$. Por exemplo, pacientes com TOC podem apresentar insight reduzido em relação ao principal sintoma obsessivo-compulsivo, aos pensamentos obsessivos e aos rituais compulsivos em geral, às dimensões específicas de sintomas obsessivo-compulsivos, ou mesmo à própria existência do TOC como um todo. A maioria dos estudos define o seu objeto de insight de forma arbitrária e idiossincrásica. Pior, ao falar de TOC com pouco insight, muitos estudos não esclarecem a qual objeto de insight se referem exatamente.

Em segundo lugar, embora os achados fenotípicos associados a baixo insight em estudos categoriais e dimensionais sejam muito variados, uma análise mais detalhada revela que existe certa convergência entre eles ${ }^{47}$. Este fenômeno não chega a ser surpreendente, uma vez que, em uma parcela significativa dos estudos, o "empobrecimento do insight" (variável categórica) é determinado por um ponto de corte arbitrário no escore final de um instrumento dimensional que mede o insight.

Neste sentido, estudos que tratam o insight no TOC como variável categórica tendem a encontrar mais frequentemente atributos contínuos (como idade de início, tempo de doença, gravidade de sintomas, extensão do comprometimento funcional e familiar e magnitude de resposta ao tratamento) como características associadas ao TOC de pouco insight. Por outro lado, estudos que abordam o insight no TOC como uma variável contínua tendem a descrever, com maior frequência, pior insight na presença de certos atributos categoriais (como o tipo de sintoma obsessivo-compulsivo, padrão de comorbidades, curso, história familiar e ausência de resposta terapêutica). Desta forma, podemos afirmar que parte da heterogeneidade dos achados nos estudos sobre o insight no TOC reflete apenas o tipo de abordagem adotada e que a redução dos níveis de insight no TOC é um fenômeno quase que universalmente associado a um quadro mais grave, quaisquer que sejam as variáveis contempladas.

Finalmente, caso existisse um subtipo de TOC caracterizado por pouco insight, deveriam ser observadas "zonas de raridade" 48 , i.e. sintomas ou características não compartilhados por pacientes com muito e pouco insight. Em nossa revisão, não fomos capazes de identificar estudos que se propusessem a investigar a existência desse fenômeno no TOC, uma estratégia taxométrica que demandaria o emprego de análises estatísticas mais complexas (e.g. MAXCOV-HITMAX). Na realidade, a existência de "zonas de raridade" entre diferentes síndromes segue sendo um ponto ainda controverso em diferentes transtornos psiquiátricos, e.g esquizofrenia vs. transtorno bipolar ${ }^{48}$ e depressão uni vs. bipolar ${ }^{49}$.

Quaisquer que sejam as abordagens adotadas (categórica vs. dimensional), as implicaçôes de uma boa caracterização do insight para o diagnóstico diferencial e manejo terapêutico de pacientes com TOC ainda não foram suficientemente estudadas. Por exemplo, o clínico pode encontrar dificuldades para diferenciar o TOC com pouco ou nenhum insight do transtorno de personalidade obsessivo-compulsivo, no qual os sintomas são também ego-sintônicos ${ }^{50}$. Além disso, o TOC com pouco ou nenhum insight pode estar associado a níveis mais elevados de acomodação familiar, fator este que pode mediar também a ausência de resposta ao tratamento ${ }^{51,52}$. Finalmente, estudos sugerem que insight do paciente com TOC melhora com o tratamento farmacoterápico convencional com IRS ${ }^{10}$, restando a impressão ainda não testada de que o mesmo fenômeno pode ser observado após o emprego de abordagens psicoeducativas e psicoterápicas $^{53}$.

Cabe enfatizar a falta de uma visão do insight como fenômeno dependente também de fatores ambientais, como aspectos culturais, religiosos e sociais, características que estão imersas nas crenças de cada indivíduo e que, por isso, influenciam na percepção sobre si mesmo e, consequentemente, sobre a sua morbidade. Em outras palavras, níveis particularmente reduzidos de insight deveriam ser esperados também em 1) pacientes escandinavos com predomínio de sintomas de simetria/organização, 2) pacientes muçulmanos ou judeus ortodoxos com predomínio de sintomas sexuais/religiosos e 3) pacientes provenientes de grandes centros brasileiros com predomínio de sintomas de agressão/checagem. Infelizmente, não encontramos estudos que levassem essas variáveis em consideração.

Estudos futuros, que utilizem conceitos mais padronizados de insight e investiguem os correlatos ambientais e biológicos destas características, devem ser desenvolvidos para que se possa avançar na condução terapêutica deste grupo de pacientes graves, ainda tão pouco compreendido. 
Financiamento e conflito de interesse

\begin{tabular}{|c|c|c|c|c|c|c|c|}
\hline $\begin{array}{l}\text { Membro do } \\
\text { grupo de } \\
\text { autores }\end{array}$ & $\begin{array}{l}\text { Local de } \\
\text { trabalho }\end{array}$ & $\begin{array}{l}\text { Verba de } \\
\text { pesquisa }^{1}\end{array}$ & $\begin{array}{c}\text { Outro apoio à pesquisa } \\
\text { ou educação médica } \\
\text { continuada }\end{array}$ & $\begin{array}{c}\text { Honorários } \\
\text { de } \\
\text { palestrante }\end{array}$ & $\begin{array}{c}\text { Participação } \\
\text { acionária }\end{array}$ & $\begin{array}{l}\text { Consultorl } \\
\text { conselho } \\
\text { consultivo }\end{array}$ & Outro $^{3}$ \\
\hline $\begin{array}{l}\text { Júlia M. } \\
\text { Fontenelle }\end{array}$ & IPUB/UFRJ & - & - & - & - & - & - \\
\hline $\begin{array}{l}\text { Lívia da S. } \\
\text { Santana }\end{array}$ & IPUB/UFRJ & - & - & - & - & - & - \\
\hline $\begin{array}{l}\text { Larissa da R. } \\
\text { Lessa }\end{array}$ & IPUB/UFRJ & - & - & - & - & - & - \\
\hline $\begin{array}{l}\text { Mara S. da } \\
\text { Victoria }\end{array}$ & IPUB/UFRJ & - & - & - & - & - & - \\
\hline $\begin{array}{l}\text { Mauro V. } \\
\text { Mendlowicz }\end{array}$ & IPUB/UFRJ & - & - & - & - & - & - \\
\hline $\begin{array}{l}\text { Leonardo F. } \\
\text { Fontenelle }\end{array}$ & IPUB/UFRJ & $\begin{array}{c}\text { CNPq }^{\star \star \star *} \\
\text { FAPERJ J** }^{*}\end{array}$ & $\begin{array}{l}\text { Bolsa Produtividade } \\
\text { CNPq }^{*}\end{array}$ & Eli-Lilly* & - & - & $\begin{array}{c}\text { Lundbeck }^{*} \\
\text { Servier* } \\
\text { Editora } \\
\text { Guanabara- } \\
\text { Koogan }\end{array}$ \\
\hline
\end{tabular}

* Modesto

** Significativa

*** Significativa. Montantes fornecidos à instituição do autor ou a colega onde o autor tem participação, não diretamente ao autor

Nota: IPUB/UFRJ = Instituto de Psiquiatria, Universidade Federal do Rio de Janeiro;

$C N P q=$ Conselho Nacional de Desenvolvimento Cientifico e Tecnológico; FAPERJ =

Fundação de Amparo à Pesquisa do Estado do Rio de Janeiro.

Mais informações consultar as instruções aos autores

\section{Referências}

1. Sims A. Symptoms in the mind: an introduction to descriptive psychopathology. 3rd edi. London: Saunders; 2006.

2. Insel TR, Akiskal HS. Obsessive-compulsive disorder with psychotic features: a phenomenologic analysis. Am J Psychiatry. 1986;143(12):1527-33.

3. Solyom L, DiNicola VF, Phil M, Sookman D, Luchins D. Is there an obsessive psychosis? Aetiological and prognostic factors of an atypical form of obsessive-compulsive neurosis. Can J Psychiatry. 1985;30(5):372-80.

4. Ballerini A, Stanghellini G. Phenomenological questions about obsession and delusion. Psychopathology. 1989;22(6):315-9.

5. Kozak MJ, Foa EB. Obsessions, overvalued ideas, and delusions in obsessivecompulsive disorder. Behav Res Ther. 1994;32(3):343-53.

6. O'Dwyer AM, Marks I. Obsessive-compulsive disorder and delusions revisited. Br J Psychiatry. 2000;176:281-4.

7. Rachman S. Some similarities and differences between obsessional ruminations and morbid preoccupations. Can Psychiatr Assoc J. 1973;18(1):71-4.

8. Türksoy N, Tükel R, Ozdemir O, Karali A. Comparison of clinical characteristics in good and poor insight obsessive-compulsive disorder. J Anxiety Disord. 2002;16(4):413-23.

9. Eisen JL, Phillips KA, Baer L, Beer DA, Atala KD, Rasmussen SA. The Brown Assessment of Beliefs Scale: reliability and validity. Am J Psychiatry. 1998;155(1):102-8.

10. Matsunaga H, Kiriike N, Matsui T, Oya K, Iwasaki Y, Koshimune K, Miyata A, Stein DJ. Obsessive-compulsive disorder with poor insight. Compr Psychiatry. 2002;43(2):150-7.

11. Bellino S, Patria L, Ziero S, Bogetto F. Clinical picture of obsessive-compulsive disorder with poor insight: a regression model. Psychiatry Res. 2005;136(23):223-31.

12. Foa EB, Kozak MJ, Goodman WK, Hollander E, Jenike MA, Rasmussen SA. DSM-IV field trial: obsessive-compulsive disorder. Am J Psychiatry. 1995;152(1):90-6.

13. Eisen JL, Rasmussen SA. Obsessive compulsive disorder with psychotic features. J Clin Psychiatry. 1993;54(10):373-9.

14. Marazziti D, Dell'Osso L, Di Nasso E, Pfanner C, Presta S, Mungai F, Cassano GB. Insight in obsessive compulsive disorder: a study of an Italian sample. Eur Psychiatry. 2002;17(7):407-10.

15. Catapano F, Sperandeo R, Perris F, Lanzaro M, Maj M. Insight and resistance in patients with obsessive compulsive disorder. Psychopathology. 2001;34(2):62-8.
16. Ravi Kishore V, Samar R, Janardhan Reddy YC, Chandrasekhar CR, Thennarasu K. Clinical characteristics and treatment response in poor and good insight obsessive-compulsive disorder. Eur Psychiatry. 2004;19(4):202-8.

17. Alonso $P$, Menchón JM, Segalàs $C$, Jaurrieta N, Jiménez-Murcia $S$, Cardoner N, Labad J, Real E, Pertusa A, Vallejo J. Clinical implications of insight assessment in obsessive-compulsive disorder. Compr Psychiatry. 2008;49(3):305-12.

18. Storch EA, Milsom VA, Merlo LJ, Larson M, Geffken GR, Jacob ML, Murphy TK, Goodman WK. Insight in pediatric obsessive-compulsive disorder: associations with clinical presentation. Psychiatry Res. 2008;160(2):21220.

19. De Berardis D, Campanella D, Serront N, Gambi F, Carano A, La Rovere R, Nardella E, Pizzorno AM, Cotellassa C, Salerno RM, Ferro FM. Insight and perceived expressed emotion among adult outpatients with obsessivecompulsive disorder. I Psychiatr Pract. 2008;14(3):154-9.

20. Hantouche EG. Syndrome de Lenteur Obsessionnelle (SLO): Nature, fréquence et influence sur l'expression clinique et la réponse au traitement dans le trouble obsessionnel-compulsif. Ann Méd Psychol. 2000;158(1):33-42.

21. Abramowitz JS, Brigidi BD, Foa EB. Health concerns in patients with obsessivecompulsive disorder. J Anxiety Disord. 1999;13(5):529-39.

22. Tumkaya S, Karadag F, Oguzhanoglu NK, Tekkanat C, Varma G, Ozdel O, Ate çi F. Schizophrenia with obsessive-compulsive disorder and obsessivecompulsive disorder with poor insight: a neuropsychological comparison. Psychiatry Res. 2009;165(1-2):38-46.

23. Aigner M, Zitterl W, Prayer D, Demal U, Bach M, Prayer L, Stompe T, Lenz G. Magnetic resonance imaging in patients with obsessive-compulsive disorder with good versus poor insight. Psychiatry Res. 2005;140(2):173-9.

24. Belloch A, Del Valle G, Morillo C, Carrió C, Cabedo E. To seek advice or not to seek advice about the problem: the help-seeking dilemma for obsessive-compulsive disorder. Soc Psychiatry Psychiatr Epidemiol. 2009; 44(4):257-64.

25. Himle JA, Van Etten ML, Janeck AS, Fischer DJ. Insight as a Predictor of Treatment Outcome in Behavioral Group Treatment for ObsessiveCompulsive Disorder. Cognitive Ther Res. 2006;30(5):661-6.

26. Hantouche EG, Bouhassira M, Lancrenon S. Prospective follow-up over a 12 month period of a cohort of 155 patients with obsessivecompulsive disorder: phase III National DRT-TOC Study. Encephale. 2000;26(6):73-83 
27. Erzegovesi S, Cavallini MC, Cavedini P, Diaferia G, Locatelli M, Bellodi L. Clinical predictors of drug response in obsessive-compulsive disorder. $J$ Clin Psychopharmacol. 2001;21(5):488-92.

28. De Berardis D, Campanella D, Gambi F, Sepede G, Salini G, Carano A, La Rovere R, Pelusi L, Penna L, Cicconetti A, Cotellessa C, Salerno RM, Ferro FM. Insight and alexithymia in adult outpatients with obsessive-compulsive disorder. Eur Arch Psychiatry Clin Neurosci. 2005;255(5):350-8.

29. Eisen JL, Phillips KA, Coles ME, Rasmussen SA. Insight in obsessive compulsive disorder and body dysmorphic disorder. Compr Psychiatry. 2004; 45(5):10-5.

30. Eisen JL, Mancebo MA, Pinto A, Coles ME, Pagano ME, Stout R, Rasmussen SA. Impact of obsessive-compulsive disorder on quality of life. Compr Psychiatry. 2006;47(4):270-5.

31. Tolin DF, Abramowitz JS, Kozak MJ, Foa EB. Fixity of belief, perceptual aberration, and magical ideation in obsessive-compulsive disorder. $J$ Anxiety Disord. 2001;15(6):501-10

32. Matsunaga H, Kiriike N, Matsui T, Iwasaki Y, Koshimune K, Ohya K, Stein DJ. A comparative study of clinical features between pure checkers and pure washers categorized using a lifetime symptom rating method. Psychiatry Res. 2001;105(3):221-9.

33. Storch EA, Lack CW, Merlo LJ, Geffken GR, Jacob ML, Murphy TK, Goodman WK. Clinical features of children and adolescents with obsessive-compulsive disorder and hoarding symptoms. Compr Psychiatry. 2007:48(4):313-8.

34. Lochner C, Hemmings SM, Kinnear CJ, Niehaus DJ, Nel DG, Corfield VA, Moolman-Smook JC, Seedat S, Stein DJ. Cluster analysis of obsessive-compulsive spectrum disorders in patients with obsessivecompulsive disorder: clinical and genetic correlates. Compr Psychiatry. 2005;46(1):14-9

35. Marazziti D, Giannotti D, Catena MC, Carlini M, Dell'Osso B, Presta S, Pfanner C, Mungai F, Dell'Osso L. Insight in body dysmorphic disorder with and without comorbid obsessive-compulsive disorder. CNS Spectr. 2006;11(7):494-8

36. Nakata AC, Diniz JB, Torres AR, de Mathis MA, Fossaluza V, Bragancas CA, Ferrão Y, Miguel EC. Level of insight and clinical features of obsessivecompulsive disorder with and without body dysmorphic disorder. CNS Spectr. 2007;12(4):295-303.

37. Poyurovsky M, Faragian S, Pashinian A, Heidrach L, Fuchs C, Weizman R, Koran L. Clinical characteristics of schizotypal-related obsessive-compulsive disorder. Psychiatry Res. 2008;159(1-2): 254-8.

38. Rodrigues Torres A, Del Porto JA. Comorbidity of obsessive-compulsive disorder and personality disorders. A Brazilian controlled study. Psychopathology. 1995;28(6):322-9.

39. Ruta L, Mugno D, D'Arrigo VG, Vitiello B, Mazzone L. Obsessive-compulsive traits in children and adolescents with Asperger syndrome. Eur Child Adolesc Psychiatry. In press 2009.

40. Ozdemir O, Tükel R, Türksoy N, Uçok A. Clinical characteristics in obsessive-compulsive disorder with schizophrenia. Compr Psychiatry. 2003;44(4):311-6.

41. Poyurovsky M, Faragian S, Kleinman-Balush V, Pashinian A, Kurs R, Fuchs C. Awareness of illness and insight into obsessive-compulsive symptoms in schizophrenia patients with obsessive-compulsive disorder. J Nerv Ment Dis. 2007;195(9):765-8.

42. Tükel R, Meteris H, Koyuncu A, Tecer A, Yazici O. The clinical impact of mood disorder comorbidity on obsessive-compulsive disorder. Eur Arch Psychiatry Clin Neurosci. 2006;256(4):240-5.

43. Hollander E, Baldini Rossi N, Sood E, Pallanti S. Risperidone augmentation in treatment-resistant obsessive-compulsive disorder: a double-blind, placebo-controlled study. Int J Neuropsychopharmacol. 2003;6(4):397401.

44. Raffin AL, Guimarães Fachel JM, Ferrão YA, Pasquoto de Souza F, Cordioli AV. Predictors of response to group cognitive-behavioral therapy in the treatment of obsessive-compulsive disorder. Eur Psychiatry. 2009;24(5):297-306.

45. Eisen JL, Rasmussen SA, Phillips KA, Price LH, Davidson J, Lydiard RB, Ninan $\mathrm{P}$, Piggott T. Insight and treatment outcome in obsessive-compulsive disorder. Compr Psychiatry. 2001;42(6):494-7.

46. Marková IS, Jaafari N, Berrios GE. Insight and obsessive-compulsive disorder: a conceptual analysis. Psychopathology. 2009;42(5): $277-82$.
47. Kraemer HC, Noda A, O’Hara R. Categorical versus dimensional approaches to diagnosis: methodological challenges. J Psychiatr Res. 2004;38(1):17-25.

48. Kendell R, Jablensky A. Distinguishing between the validity and utility of psychiatric diagnoses. Am J Psychiatry. 2003;160(1):4-12.

49. Benazzi F. Bipolar II disorder and major depressive disorder: continuity or discontinuity? World J Biol Psychiatry. 2003;4(4):166-71.

50. Bailey GR Jr. Cognitive-behavioral treatment of obsessive-compulsive personality disorder. J Psychol Pract. 1998;4(1):51-9.

51. Ferrão YA, Diniz JB, Lopes AC, Shavitt RG, Greenberg B, Miguel E. Resistance and refractoriness in obsessive-compulsive disorder. Rev Bras Psiquiatr. 2007;29(Suppl 2):S66-76.

52. Ferrão YA, Shavitt RG, Bedin NR, de Mathis ME, Carlos Lopes A, Fontenelle LF, Torres AR, Miguel EC. Clinical features associated to refractory obsessive-compulsive disorder. J Affect Disord. 2006;94(1-3):199-209.

53. Prazeres AM, Souza WF, Fontenelle LF. Cognitive-behavior therapy for obsessive-compulsive disorder: a systematic review of the last decade. Rev Bras Psiquiatr. 2007;29(3):262-70. 